Universidade Tecnológica Federal do Paraná - UTFPR

Campus Ponta Grossa - Paraná - Brasil

ISSN: 1981-3686/v. 04, n. 02: p. 141-152, 2008

DOI: $10.3895 / \mathrm{S} 1981-36862010000200003$
Revista Brasileira de Tecnologia

Agroindustrial

\title{
CARACTERIZAÇÃO FÍSICA E NUTRICIONAL DE TRÊS ESPÉCIES DE PEIXES AMAZÔNICOS
}

\section{PHYSICAL AND NUTRITIONAL CHARACTERIZATION AND LIPID PROFILE OF THREE AMAZON FISH SPECIES}

\author{
Hugo Antonio Lima de Souza ${ }^{1}$; Ádria de Sousa Bentes²; \\ Marilda Garcia Simões ${ }^{3}$; Mauro José Pantoja Fontelles ${ }^{4}$ \\ ${ }^{1}$ Universidade Estadual de Campinas - Unicamp - Campinas - Brasil hugolima@ @ea.unicamp.br; ${ }_{-}^{2}$ \\ ${ }^{2}$ Universidade Estadual de Campinas - Unicamp - Campinas - Brasil adriasb@ fea.unicamp.br \\ ${ }^{3}$ Universidade do Estado do Pará - UEPA - Belém - Brasil marildags@ uol.com.br \\ ${ }^{4}$ Universidade da Amazônia - Unama - Belém - Brasil mfontelles@bol.com.br
}

\begin{abstract}
Resumo
O Estado do Pará é o segundo maior produtor nacional de pescado e apresenta consumo três vezes superior à média brasileira. Contudo, a literatura científica atual oferece poucas informações sobre os aspectos nutricionais dos peixes da Região Amazônica. O presente estudo teve como objetivo avaliar as características físicas, químicas, determinar o perfil de ácidos graxos e avaliar a qualidade nutricional das espécies: Pescada Amarela (Cynoscion acoupa), Bagre (Arius passany) e Mapará (Hypophthalmus edentatus). Os modelos matemáticos lineares mostraram-se mais adequados que os exponenciais para prever a massa por meio do comprimento e da circunferência abdominal. Os valores de umidade, proteínas, cinzas, lipídeos, carboidratos e valor energético foram de: $79.82 \%, 16.17 \%, 1.11 \%, 0.67 \%, 2.23 \%$ e $79.63 \mathrm{kcal} / 100 \mathrm{~g}$ para Pescada Amarela; $80.58 \%, 16.66 \%, 1.06 \%, 0.52 \%, 1,18 \%$ e 76.04 kcal/100g para o Bagre; e 69.61\%, 13.89\%, 0.79\%, 14.53\%, 1,18\% e 191.05 kcal/100g para o Mapará. A Pescada Amarela apresentou o maior somatório de ácidos graxos monoinsaturados $(34,30 \%)$ e poliinsaturados $(28,75 \%)$, bem como de $\omega 3$ (29,22\%). As recomendações de EPA + DHA são atendidas pelas seguintes porções de filé cru: $595 \mathrm{~g}$ de Bagre; $167 \mathrm{~g}$ de Pescada Amarela ou $21 \mathrm{~g}$ de Mapará. A Pescada Amarela apresentou os melhores índices de qualidade nutricional, indicando a melhor composição lipídica em relação às outras espécies estudadas.
\end{abstract}

Palavras-chave: ácidos graxos, qualidade nutricional, peixes amazônicos.

\section{Introdução}

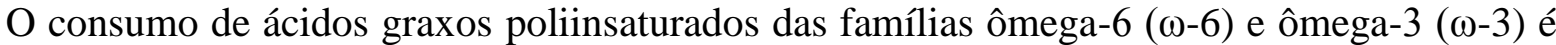
considerado essencial na dieta de mamíferos, visto que não podem ser sintetizados por esses organismos e são precursores de vários compostos importantes para o seu funcionamento, entre eles os eicosanóides (CURTIS-PRIOR, 2004; LANDS, 2005; NELSON e COX, 2005). Contudo, a dieta ocidental é demasiadamente rica em ácidos graxos da família $\omega-6$ e pobre em $\omega-3$ e em razão do 
alto consumo de gorduras vegetais ricas em $\omega-6$, este desequilíbrio pode levar a um maior risco de doenças cardiovasculares (FAO/WHO, 1994; SUGANO e HIRAHARA, 2000; SUÁREZMAHECHA et al., 2002).

Os principais índices utilizados para a avaliação da qualidade dos lipídeos são a razão entre os ácidos graxos ômega 6 e ômega $3(\Sigma \omega-6 / \Sigma \omega-3)$ e a razão de ácidos graxos poliinsaturados e

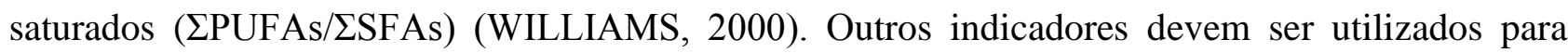
complementar esta avaliação, como os índices de aterogenicidade e trombogenicidade e a razão hipercolesterolêmicos/hipocolesterolêmicos, uma vez que cada ácido graxo saturado apresenta efeito metabólico distinto (DIETSCHY, 1998; WILLIAMS, 2000; SANTOS-SILVA et al., 2002).

O aumento do consumo de fontes de $\omega-3$ tem sido preconizado como benéfico à saúde, pois são importantes para o desenvolvimento $\mathrm{e}$ funcionamento do sistema nervoso central (NETTLETON, 1995), promovem a redução dos triglicerídeos séricos, a diminuição de alergias crônicas (SIKORSKI, 1994; SUAREZ-MAHECHA et al., 2002) e podem prevenir arteriosclerose e trombose (HUY, 1996; SHAHIDI e FINLEY, 2001). Entre as fontes alimentícias de $\omega-3$ podem ser destacados os pescados, principalmente pela presença de ácidos graxos altamente insaturados como o ácido eicosapentaenóico (C20:5) e o docosaexaenóico (C 22:6) (NETTLETON, 1995; HUI, 1996; SHAHIDI e FINLEY, 2001).

O Estado do Pará é o maior produtor de pescado da Região Amazônica e o segundo maior do Brasil, com produção de 152.830 toneladas no ano de 2006 (BRASIL, 2008). Contudo, poucos são os estudos encontrados a respeito da composição nutricional dos pescados desta região, principalmente no que tange à determinação do perfil de ácidos graxos. Neste contexto, o presente estudo teve como objetivo avaliar as características físicas e químicas, além do perfil de ácidos graxos e a qualidade nutricional dos lipídeos de três peixes comercialmente importantes da Região Amazônica Oriental: Pescada Amarela (Cynoscion acoupa), Bagre (Arius passany) e Mapará (Hypophthalmus edentatus).

\section{Material e Métodos}

Para cada espécie, foram adquiridos vinte exemplares em entreposto pesqueiro localizado no distrito de Icoaraci, no município de Belém, Pará. Os parâmetros de frescor foram analisados de acordo com Larsen et al. (1991). O estudo não considerou sexo, idade ou estágio de maturação das espécies de peixes.

Após a coleta, os peixes foram separados aleatoriamente em quatro lotes de cinco peixes para cada espécie, nos quais foram aferidos: o comprimento (medida do extremo anterior da cabeça até os raios medianos da nadadeira caudal, estando o animal estendido sobre uma superfície plana); 
a circunferência corporal (medida na região imediatamente posterior ao fim da primeira nadadeira dorsal), com o auxílio de trena, com precisão de $0,5 \mathrm{~cm}$ e a massa, em balança eletrônica com precisão de $0,05 \mathrm{Kg}$.

Os peixes foram filetados manualmente com faca em aço inoxidável e a porção superior da musculatura de um dos filés de cada exemplar foi retirada. Posteriormente, os quatro lotes de cada espécie foram homogeneizados, com o auxílio de um moinho de facas, para a obtenção de uma massa homogênea (pool amostral).

Para as análises de composição centesimal foi elaborado um novo pool amostral para cada espécie, a partir da homogeneização de alíquotas, de mesmo peso, dos 4 lotes da mesma espécie. As análises de composição química foram efetuadas em triplicata $(n=3)$ de acordo com os seguintes protocolos: umidade, pelo método gravimétrico em estufa a $105^{\circ} \mathrm{C}$; cinzas pelo método gravimétrico por calcinação em mufla a $550^{\circ} \mathrm{C}$ e proteínas pelo método de micro Kjeldahl (AOAC, 1997). Utilizou-se o fator de conversão N de 4,94 de acordo com Salo-Väänänen e Koivistoinen (1996). Os lipídeos totais foram determinados por extração com mistura de solventes a frio, conforme descrito por Bligh e Dyer (1959). Os carboidratos e o valor energético foram determinados segundo a Resolução RDC 360 (BRASIL, 2003).

A determinação da composição de ácidos graxos foi realizada para os 4 lotes de cada espécie $(\mathrm{n}=4)$. A fração lipídica total foi submetida à esterificação de acordo com o recomendado pela Comission des Communatés Européennes (1977). A separação dos ésteres metílicos foi realizada em um cromatógrafo a gás Varian, modelo CP-3380, equipado com detector de ionização de chama (FID) e coluna capilar Varian modelo CP-Sil 88 (60m x 0,25mm), utilizando hélio, como gás de arraste. A identificação dos ácidos graxos foi efetuada através da comparação dos tempos de retenção dos ésteres metílicos da amostra com os observados em uma amostra padrão (GLC - 68B e 68D, NuCheck). A quantificação dos ésteres metílicos foi realizada através da integração da área dos picos pelo software Star 6.0 (2003).

Utilizando-se os dados do perfil lipídico pôde-se determinar a qualidade nutricional da fração lipídica dos peixes estudados, a partir dos índices de aterogenicidade (IA) e trombogenicidade (IT) (ULBRICHT e SOUTHGATE, 1991) e da razão entre ácidos graxos hipocolesterolêmicos e hipercolesterolêmicos $\left(\mathrm{HH}=\sum\right.$ ác. graxos hipocolesterolêmicos/ $\sum$ ác. graxos hipercolesterolêmicos) (SANTOS-SILVA et al., 2002), expressos por meio das equações 1, 2 e 3 , respectivamente.

$$
\mathrm{IA}=\frac{[\mathrm{C} 12: 0+(4 \times \mathrm{C} 14: 0)+\mathrm{C} 16: 0]}{\left(\mathrm{SAGMI}+\sum \omega 6+\Sigma \omega 3\right)}
$$




$$
\begin{gathered}
\mathrm{IT}=\frac{(\mathrm{C} 14: 0+\mathrm{C} 16: 0+\mathrm{C} 18: 0)}{\left[\left(0,5 \times \sum \text { AGMI }\right)+\left(0,5 \times \sum \omega 6\right)+\left(3 \times \sum \omega 3\right)+\left(\sum \omega 3 / \sum \omega 6\right)\right]} \\
\mathrm{HH}=\frac{(\mathrm{C} 18: 1+\mathrm{C} 18: 2+\mathrm{C} 20: 4+\mathrm{C} 18: 3+\mathrm{C} 20: 5+\mathrm{C} 22: 5+\mathrm{C} 22: 6)}{(\mathrm{C} 14: 0+\mathrm{C} 16: 0)}
\end{gathered}
$$

Onde:

$\sum \mathrm{AGMI}=$ somatório dos ácidos graxos monoinsaturados;

$\sum \omega 6=$ somatório dos ácidos graxos $\omega 6$;

$\sum \omega 3=$ somatório dos ácidos graxos $\omega 3$.

Os resultados de caracterização física foram submetidos ao modelo linear múltiplo (Equação 4) e o modelo exponencial (Equação 5), tendo como variável resposta o peso, encontrando-se os Coeficientes de Determinação $\left(\mathrm{R}^{2}\right)$ e Coeficientes de Correlação de Pearson $(\mathrm{R})$. A comparação entre as três espécies dos resultados da caracterização física, composição química e perfil de ácidos graxos foi realizada através da Análise de Variância (ANOVA de um critério - teste F) para verificação de diferenças estatisticamente significativas entre elas. Havendo diferenças entre as espécies, o Teste ANOVA foi complementado pelo teste Tukey.

As análises estatísticas foram processadas com o auxílio do software STATISTICA for windows 5.5 (2000).

$$
\begin{gathered}
\text { Peso }=\mathrm{a}+\mathrm{b} * \text { Comprimento }+\mathrm{c} * \text { Circunferência }^{\text {Peso }}=\mathrm{a} * \text { Comprimento }^{\mathrm{b}} \\
\text { Pemping }
\end{gathered}
$$

\section{Resultados e discussão}

As espécies estudadas apresentaram diferenças significativas nos aspectos físicos estudados (Tabela 1), com exceção da circunferência corporal para as espécies Pescada Amarela e Bagre.

Tabela 1 - Análise biométrica das espécies estudadas*

\begin{tabular}{cccc}
\hline & Pescada Amarela & Bagre & Mapará \\
\hline Massa (kg) & $7,4 \pm 1,85^{\mathrm{a}}$ & $4,4 \pm 0,88^{\mathrm{b}}$ & $0,5 \pm 0,07^{\mathrm{c}}$ \\
Comprimento (m) & $0,9 \pm 0,07^{\mathrm{a}}$ & $0,8 \pm 0,05^{\mathrm{b}}$ & $0,5 \pm 0,02^{\mathrm{c}}$ \\
Circunferência corporal (m) & $0,5 \pm 0,05^{\mathrm{a}}$ & $0,4 \pm 0,03^{\mathrm{a}}$ & $0,2 \pm 0,01^{\mathrm{b}}$ \\
\hline
\end{tabular}

* Média \pm desvio padrão; $\mathrm{n}=20$. Letras diferentes na mesma linha indicam diferença significativa entre as espécies (p < 0,05) calculada por meio do Teste de Tukey.

A regressão linear múltipla indicou que o comprimento e a circunferência corporal apresentaram correlação positiva com a massa (Figura 1), sendo esta relação muito forte para a Pescada Amarela $(\mathrm{R}=0,92)$ e Bagre $(\mathrm{R}=0,96)$, e forte para o Mapará $(\mathrm{R}=0,81)$. O modelo linear apresentou bom ajuste para a Pescada Amarela $\left(R^{2}=0,85\right)$ e Bagre $\left(R^{2}=0,93\right)$, contudo não 
apresentou bom ajuste para o Mapará $\left(\mathrm{R}^{2}=0,65\right)$. Entretanto, os valores de $\mathrm{p}$ foram adequados para todos os modelos $(\mathrm{p}<0,002)$.

Figura 1 - Regressão linear múltipla do peso em função do comprimento e circunferência corporal.

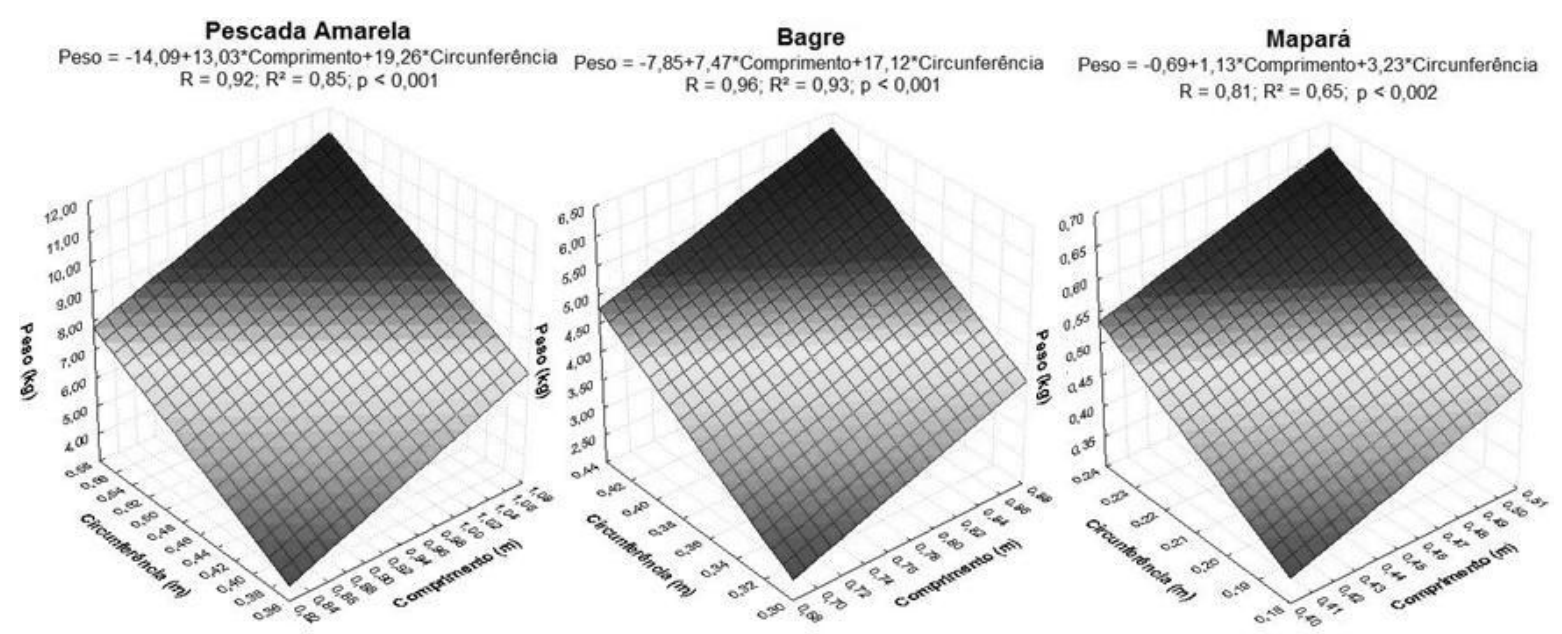

O modelo exponencial mostrou-se menos adequado que o linear para as espécies estudadas, com R² de 0,78; 0,84 e 0,47 para a Pescada Amarela, Bagre e Mapará, respectivamente. Porém, outros trabalhos indicam melhores ajustes $\mathrm{R}^{\mathbf{2}}>0,84$ para Saramunete (Pseudupeneus maculatus Bloch, 1793) e $\mathrm{R}^{2}>$ 0,94 para Pargo, (Lutjanus purpureus Poey, 1875) (CAMPOS e OLIVEIRA, 2001; SOUZA et al., 2006).

Os resultados da composição química (Tabela 2) indicam que as três espécies apresentaram diferenças significativas entre si, em, no mínimo, um atributo. As espécies Pescada Amarela e Bagre diferiram apenas na umidade e a espécie Mapará diferiu desses em toda composição química.

Tabela 2 - Composição centesimal das três espécies estudadas em g/100g de filé

\begin{tabular}{|c|c|c|c|}
\hline & Pescada Amarela & Bagre & Mapará \\
\hline Umidade* $^{*}$ & $79,8 \pm 0,13^{\mathrm{a}}$ & $80,6 \pm 0,22^{b}$ & $69,6 \pm 0,22^{c}$ \\
\hline Proteínas* & $16,1 \pm 0,14^{\mathrm{a}}$ & $16,7 \pm 0,49^{\mathrm{a}}$ & $13,9 \pm 0,41^{\mathrm{b}}$ \\
\hline Cinzas* & $1,1 \pm 0,12^{\mathrm{a}}$ & $1,1 \pm 0,09^{\mathrm{a}}$ & $0,8 \pm 0,02^{\mathrm{b}}$ \\
\hline Lipídeos* & $0,7 \pm 0,16^{\mathrm{a}}$ & $0,5 \pm 0,11^{\mathrm{a}}$ & $14,5 \pm 0,25^{\mathrm{b}}$ \\
\hline Carboidratos & 2,2 & 1,2 & 1,2 \\
\hline Valor energético (kcal/100g) & 79,6 & 76,0 & 191,0 \\
\hline
\end{tabular}

O valor de umidade variou de 69,6\%, no Mapará, a 806\%, no Bagre. O Mapará também apresentou o menor teor de proteínas $(13,9 \%)$ e de cinzas $(0,8 \%)$. O tecido muscular do Mapará apresentou maior teor médio de lipídeos (14,5\%) e, de acordo com Ackman (1989), pode ser considerada uma espécie altamente gorda, pois a taxa de lipídeos encontrada foi maior que $8 \%$, 
enquanto as espécies Bagre e Pescada Amarela apresentaram valores inferiores a 2\%, o que as caracteriza como espécies magras. De acordo com classificação de Sanches (1989) a Pescada Amarela e o Bagre são peixes do tipo A, pois apresentam alto teor de proteínas e baixo teor de lipídeos, enquanto o Mapará é um peixe do tipo C, pois apresenta baixo teor de proteínas e alto teor de lipídeos.

O teor de lipídeos observado no Mapará foi de cerca de quatro vezes o encontrado por Franco (1999), contudo dentro de intervalo encontrado por Oliveira et al. (2003) (8,97 a 18,99\%). Fernandes et al. (2007) encontraram 0,63\% de lipídeos no Bagre Rosado (Tachysurus barbus) e 0,99\% na Pescada Amarela.

Os teores de carboidratos encontrados foram baixos para as três espécies, comum em peixes. De acordo com Ogawa e Maia (1999) o conteúdo de carboidratos em peixe é de 0,3 a 1\%, dos quais os mais importantes são glicogênio e mucopolissacarídeos.

Os valores energéticos da Pescada Amarela e do Bagre foram próximos (79,6 kcal/100g e $76,0 \mathrm{kcal} / 100 \mathrm{~g}$ ). Entretanto, o Mapará apresentou valor energético $140 \%$ e $150 \%$ maior do que os valores encontrados para a Pescada Amarela e para o Bagre, respectivamente, reflexo do elevado conteúdo lipídico do Mapará.

Os perfis de ácidos graxos encontrados no tecido muscular das três espécies de peixes estão apresentados nas Tabelas 3 e 4, nas quais é possível se observar que os principais ácidos graxos presentes na Pescada Amarela foram o palmítico (C16:0) e o DHA (C22:6).

Tabela 3 - Composição de ácidos graxos (\%) de lipídeos totais das três espécies estudadas*

\begin{tabular}{|c|c|c|c|c|}
\hline Ácido Graxo & Nome Comum & Pescada Amarela & Bagre & Mapará \\
\hline C 14:0 & Mirístico & $3,9 \pm 0,40^{\mathrm{a}}$ & $4,6 \pm 1,48^{\mathrm{ab}}$ & $6,5 \pm 1,13^{b}$ \\
\hline C $14: 1$ & Miristoléico & $0,5 \pm 0,05^{\mathrm{a}}$ & $0,4 \pm 0,08^{a}$ & $1,4 \pm 0,03^{b}$ \\
\hline C $16: 0$ & Palmítico & $27,6 \pm 1,23^{\mathrm{a}}$ & $38,2 \pm 0,68^{b}$ & $36,7 \pm 0,40^{b}$ \\
\hline C $16: 1$ & Palmitoléico & $11,0 \pm 1,14^{\mathrm{a}}$ & $6,2 \pm 0,04^{b}$ & $7,8 \pm 1,59^{a}$ \\
\hline C $18: 0$ & Esteárico & $5,2 \pm 0,98^{a}$ & $10,4 \pm 0,39^{b}$ & $8,8 \pm 2,40^{\mathrm{a}}$ \\
\hline C $18: 1$ & Oléico & $11,9 \pm 1,27$ & $13,2 \pm 1,82$ & $11,4 \pm 0,42$ \\
\hline C $18: 1 \mathrm{~V}$ & Vacénico & $3,2 \pm 0,60$ & $3,2 \pm 0,08$ & $3,1 \pm 0,08$ \\
\hline C $18: 2$ & Linoleico & $1,5 \pm 0,21^{\mathrm{a}}$ & $3,3 \pm 0,02^{b}$ & $3,4 \pm 0,04^{\mathrm{b}}$ \\
\hline C 20:0 & Araquídico & $0,3 \pm 0,10$ & - & $0,3 \pm 0,12$ \\
\hline C 20:1 & Gadoléico & $0,8 \pm 0,16$ & $1,2 \pm 0,16$ & $1,0 \pm 0,19$ \\
\hline C $18: 3$ & Linolênico & $0,4 \pm 0,06^{\mathrm{a}}$ & $5,0 \pm 0,57^{b}$ & $5,3 \pm 0,62^{b}$ \\
\hline C $20: 2$ & EDA & $0,1 \pm 0,01$ & $0,3 \pm 0,21$ & $0,3 \pm 0,08$ \\
\hline C $20: 3$ & ETA & - & - & $0,4 \pm 0,19$ \\
\hline C 20:4 & Araquidônico & - & - & $0,5 \pm 0,05$ \\
\hline C 22:0 & Behênico & - & - & - \\
\hline C 22:1 & Erúcico & $6,4 \pm 0,82^{\mathrm{a}}$ & $3,0 \pm 0,12^{b}$ & $3,0 \pm 0,06^{\mathrm{b}}$ \\
\hline C 20:5 & EPA & $6,6 \pm 0,65^{a}$ & $4,5 \pm 0,59^{b}$ & $4,7 \pm 0,56^{\mathrm{b}}$ \\
\hline C 24:0 & Lignocérico & - & - & - \\
\hline C $24: 1$ & Nervônico & $0,8 \pm 0,05^{\mathrm{a}}$ & - & $0,4 \pm 0,01^{\mathrm{b}}$ \\
\hline C 22:6 & DHA & $20,1 \pm 4,17^{\mathrm{a}}$ & $5,3 \pm 0,46^{b}$ & $5,1 \pm 0,20^{b}$ \\
\hline
\end{tabular}

* Média \pm desvio padrão; $\mathrm{n}=4 . \mathrm{AG}=$ Ácido graxo; $\mathrm{EDA}=$ Ácido eicosadienócio; $\mathrm{EPA}=$ Á. Eicosapentaenóico; $\mathrm{ETA}=$ Á. Eicosatrienóico; $\mathrm{DHA}=$ Á. Docosahexaenóico. Letras diferentes nas colunas indicam diferença significativa entre as amostras ( $p<0,05)$-Tukey. Ausência de letras nas colunas indica diferença não significativa entre as amostras $(p>0,05)$ 
Visentainer et al. (2007) observaram que estes ácidos são majoritários também em outra espécie do mesmo gênero da Pescada Amarela, a Goete (Cynoscion jamaicensis). Nas espécies Bagre e Mapará os principais ácidos graxos foram o palmítico, seguido do oléico (C18:1). Inhamuns e Franco (2001) obtiveram resultados similares em Mapará.

Tabela 4 - Somatório das classes de ácidos graxos (\%) de lipídeos totais das três espécies estudadas*

\begin{tabular}{|c|c|c|c|}
\hline & Pescada Amarela & Bagre & Mapará \\
\hline$\overline{\Sigma \text { SFAs }}$ & $36,9 \pm 2,48^{a}$ & $53,5 \pm 1,67^{b}$ & $52,4 \pm 1,99^{b}$ \\
\hline ¿MUFAs & $34,3 \pm 1,61^{\mathrm{a}}$ & $27,5 \pm 1,89^{b}$ & $28,1 \pm 0,98^{b}$ \\
\hline$\Sigma$ PUFAs & $28,8 \pm 4,12^{\mathrm{a}}$ & $18,8 \pm 0,75^{b}$ & $19,5 \pm 1,00^{b}$ \\
\hline$\Sigma \mathrm{AG} \omega 3$ & $29,2 \pm 0,85^{\mathrm{a}}$ & $14,7 \pm 1,63^{b}$ & $15,0 \pm 1,37^{b}$ \\
\hline$\Sigma A G \omega 6$ & $1,4 \pm 0,13^{\mathrm{a}}$ & $3,6 \pm 0,34^{\mathrm{b}}$ & $3,8 \pm 0,09^{b}$ \\
\hline
\end{tabular}

* Média \pm desvio padrão; $\mathrm{n}=4$.MUFA $=$ Á. graxo monoinsaturado; PUFA $=$ Á. graxo polinstaturado; SFA = Á. graxo saturado; $\Sigma=$ Soma.Letras diferentes nas colunas indicam diferença significativa entre as amostras $(\mathrm{p}<0,05)$-Tukey. Ausência de letras nas colunas indica diferença não significativa entre as amostras $(\mathrm{p}>0,05)$

O DHA (C22:6) foi o ácido graxo ômega-3 encontrado em maior proporção na Pescada Amarela e Bagre, entretanto no Mapará o principal ácido graxo ômega-3 foi o linolênico (18:3).

A American Heart Association recomenda a ingestão diária de, no mínimo, 300 mg de EPA + DHA (KRAUSS et al., 2000; KRIS-ETHERTON et al., 2002). Com base neste dado, a ingestão diária recomendada, em gramas de filé cru, será de: 595 g de Bagre; 167 g de Pescada Amarela ou 21 g de Mapará. Bentes et al. (2009) verificaram que o consumo de 248 g de filé cru de Gurijuba, 234 g de Piramutaba ou 187 g de Dourada seriam suficientes para atender essa recomendação.

A qualidade nutricional do perfil lipídico, avaliada por diferentes índices encontra-se descrita na Tabela 5 .

Tabela 5 - Índices da qualidade nutricional do perfil lipídico das espécies estudadas*

\begin{tabular}{cccc}
\hline & Pescada Amarela & Bagre & Mapará \\
\hline$\Sigma \omega 6 / \Sigma \omega 3$ & $0,1 \pm 0,00^{\mathrm{a}}$ & $0,3 \pm 0,05^{\mathrm{b}}$ & $0,3 \pm 0,03^{\mathrm{b}}$ \\
$\Sigma$ PUFAs/ $\Sigma$ SFAs & $0,9 \pm 0,04^{\mathrm{a}}$ & $0,4 \pm 0,03^{\mathrm{b}}$ & $0,4 \pm 0,03^{\mathrm{b}}$ \\
IA & $0,7 \pm 0,02^{\mathrm{a}}$ & $1,2 \pm 0,15^{\mathrm{b}}$ & $1,3 \pm 0,02^{\mathrm{b}}$ \\
IT & $0,3 \pm 0,01^{\mathrm{a}}$ & $0,8 \pm 0,10^{\mathrm{b}}$ & $0,8 \pm 0,09^{\mathrm{b}}$ \\
HH & $1,4 \pm 0,05^{\mathrm{a}}$ & $0,7 \pm 0,09^{\mathrm{b}}$ & $0,7 \pm 0,01^{\mathrm{b}}$ \\
\hline
\end{tabular}

* Média \pm desvio padrão; $\mathrm{n}=4$. Letras diferentes nas colunas indicam diferença significativa entre as amostras $(\mathrm{p}<0,05)$-Tukey.

Dietas que apresentam valores inferiores a 4,0 são recomendadas para a diminuição dos riscos cardiovasculares, segundo o Department of Health and Social Security da Inglaterra (1984). A razão $\omega 6 / \omega 3$ foi de 0,05 na Pescada Amarela e 0,25 no Bagre e no Mapará. Visentainer et al. (2007) encontraram valores de 0,07 a 0,30 em peixes marinhos da costa brasileira, enquanto Ramos Filho et al. (2008) e Moreira et al. (2001) encontraram valores de 0,95 a 3,65 e 1,14 a 8,79, respectivamente, em peixes de água doce. 
Para a relação entre ácidos graxos polinsaturados e saturados (PUFA/SFA) são indicados valores maiores que 0,45, segundo o Department of Health and Social Security (1984). Dentre as espécies estudadas apenas a Pescada Amarela apresentou razão superior ao recomendado $(0,86)$. Também foram observados valores inferiores ao recomendado em peixes do gênero Brycon (MOREIRA et al., 2001), na Cachara (Pseudoplatystoma fasciatum) e no Pacu (Piractus mesopotamicus) (RAMOS FILHO et al., 2008).

Para os índices de aterogenicidade (IA) e trombogenicidade (IT) são desejados valores baixos (ULBRICHT e SOUTHGATE, 1991), enquanto para a razão hipocolesterolêmicos e hipercolesterolêmico $(\mathrm{HH})$ valores mais elevados são mais adequados nutricionalmente (SANTOSSILVA et al., 2002). Os IA e IT encontrados na Pescada Amarela (0,66 e 0,28) foram inferiores aos encontrados no Bagre (1,21 e 0,84) e no Mapará (1,34 e 0,80). Rueda et al. (2001) encontraram valores menores destes índices em peixes criados em cativeiro, em comparação aos selvagens, no sargo-bicudo [Diplodus puntazzo (Cetti, 1777)]. A razão de HH na Pescada Amarela $(1,36)$ foi superior ao encontrado no Bagre $(0,74)$ e no Mapará $(0,70)$, não havendo diferença estatisticamente significativa entre as duas últimas para nenhum dos índices de qualidade, indicando a maior qualidade nutricional dos lipídeos da Pescada Amarela em relação às outras espécies estudadas.

Ramos Filho et al. (2008) estudaram o IA, IT e HH de quatro espécies da região pantaneira e observaram os seguintes resultados: 0,54, 0,59 e 1,75 para o Cachara (Pseudoplatystoma fasciatum); 0,49, 0,33 e 1,84 para o Pintado (Pseudoplatystoma coruscans); 0,86, 1,16 e 1,66 para o Pacu (Piaractus mesopotamicus) e 0,70, 0,35 e 1,49 para o dourado (Salminus maxillosus).

\section{Conclusão}

A Pescada Amarela e o Bagre apresentaram boa relação protéico/calórica, enquanto o Mapára pode ser considerado uma boa fonte energética devido ao seu elevado teor de lipídios totais. As espécies Mapará e Bagre apresentaram altos teores de ácidos graxos monoinsaturados, enquanto a Pescada Amarela apresentou maior porção de ácidos graxos insaturados, principalmente os da família $\omega 3$. A espécie Pescada Amarela apresentou os melhores índices da avaliação da qualidade nutricional, mostrando-se adequada para uma dieta saudável e equilibrada, sendo excelente para a saúde humana. Apesar de apresentar índices de qualidade nutricional menos adequados que a Pescada Amarela, a espécie Mapará apresenta grande potencial como matéria prima para a produção de óleo de peixe, dado seu baixo custo, alto teor de lipídeos e elevado teor de $\omega 3$ por grama de filé cru. 


\title{
Agradecimento
}

À Pró-reitoria de Pesquisa e Pós-graduação da Universidade do Estado do Pará pelo apoio financeiro.

\begin{abstract}
Pará state is the second in the rank of Brazilian capture fisheries production, and the regional consumption is higher than triple of national mean, the current bibliography offer small quantity of information about nutritional aspects of Amazonian fishes. The aim of this work were evaluate physically, chemically, the fatty acids profile and nutritional quality of three fish species: Akoupa (Cynoscion acoupa), Passany sea catfish (Arius passany), and Maparate (Hypophthalmus edentatus). The linear mathematics models shown to be more suitable than exponentials models to predict the weight by length and abdominal circumference. The moisture, proteins, ash, fat, carbohydrate values and energetic value were: $79.82 \%, 16.17 \%, 1.11 \%, 0.67 \%, 2,23 \%$ and 79,63 $\mathrm{kcal} / 100 \mathrm{~g}$ on Akoupa; $80.58 \%, 16.66 \%, 1.06 \%, 0.52 \%, 1,18 \%$ and $76,04 \mathrm{kcal} / 100 \mathrm{~g}$ on Passany; and $69.61 \%, 13.89 \%, 0.79 \%, 14.53 \%, 1,18 \%$ and $191,05 \mathrm{kcal} / 100 \mathrm{~g}$ on Maparate. The Akoupa showed the higher sum of monounsaturated $(34.30 \%)$ and polyunsaturated fatty acids $(28.75 \%)$, as well as $\omega 3(29.22 \%)$. The EPA + DHA recommendations are attend by the follow filet crude portions: 595 $\mathrm{g}$ of Passany; $167 \mathrm{~g}$ of Akoupa or $21 \mathrm{~g}$ of Maparate. The Akoupa had the better nutritional quality index, indicating the higher nutritional quality of its fat than the others studied species.
\end{abstract}

Key-words: fatty acids, nutritional quality, Amazonian fishes.

\section{Referências}

ACKMAN, R. G. Nutritional Composition of fats in seafoods. Progress in Food and Nutrition Science, n. 13, v. 03 04, p. 161-289, 1989.

AOAC - ASSOCIATION OF OFFICIAL ANALYTICAL CHEMISTS. Official methods of analysis of AOAC International. 16 ed. Gaithersburg: AOAC, 1997.

BENTES, A. S.; SOUZA, H. A. L.; MENDONÇA, X. M. F. D.; SIMÕES, M. G. Caracterização física e química e perfil lipídico de três espécies de peixes amazônicos. Revista Brasileira de Tecnologia Agroindustrial, n. 02, v. 03, p. 97-108, 2009.

BLIGH, E. G.; DYER, W. J. A rapid method of total lipid extraction and purification. Canadian Journal Biochemistry Physiology, n. 37, n. 08, p. 911-917, 1959. DOI: 10.1139/059-099.

BRASIL. Agência Nacional de Vigilância Sanitária. Resolução RDC nº 360, de 23 de dezembro de 2003. Aprova o regulamento técnico sobre rotulagem nutricional de alimentos embalados, tornando obrigatória a rotulagem nutricional. Diário Oficial da União. 26 de dezembro de 2003, disponível em: <http://bvsms.saude.gov.br/bvs/saudelegis/anvisa/2003/res0360_23_12_2003.html>. Acesso em 09 nov. 2010.

BRASIL. Instituto Brasileiro do Meio Ambiente e dos Recursos Naturais Renováveis. Estatística da pesca 2006 Brasil: grandes regiões e unidades da federação. Brasília: IBAMA, 2008. 174 p.

CAMPOS, C. E. C.; OLIVEIRA, J. E. L. Caracterização biométrica e merística do saramunete, Pseudupeneus maculatus (Osteichthyes: Mullidae), em Ponta de Pedras, Pernambuco. Boletim do Instituto de Pesca, v. 27, n. 2, p. 185-189, 2001.

COMISSION DES COMMUNAUTÉS EUROPÉENNES. Méthode d'analyse communautaire à utiliser pour la determination de la teneur en acid érucique, en ce qui concerne les graines prises en charge par les organismes d'intervention. Official Journal of the European Communities L12, p.12-18, 1977.

CURTIS-PRIOR, P. The eicosanoids. West Sussex: Wiley, 2004. 655 p. 
DEPARTMENT OF HEALTH AND SOCIAL SECURITY. Diet and cardiovascular disease. Report on Health and Social Subjects, n. 28. London: HMSO, 1984.

DIETSCHY, J. M. Dietary fatty acids and the regulation of plasma low density lipoprotein cholesterol concentration. Journal of Nutrition, n. 128, sup. 02, p. 444S-448S, 1998.

FAO; WHO. Fats and oils in human nutrition: report of a joint expert consultation. FAO Food and Nutrition Paper, Roma, v. 57, 1994.

FERNANDES, A. C.; CASTRO, B. A.; LOURENÇO, L. F. H.; CORRÊA, N. C. F. Influência da maturação de Pescada Amarela (Cynoscion acoupa) e Bagre rosado (Tachysurus barbus) na caracterização físico-química de fishburg. In: VII Simpósio Latino-Americano de Ciência de Alimentos. Anais do Simpósio Latino-Americano de Ciência de Alimentos. Campinas, 2007.

FRANCO, G. Tabela de composição química dos alimentos. 9 ed. São Paulo: Atheneu, 1999.

GUTIERREZ, L. E.; SILVA, R. C. M. Fatty acid composition of commercially important fish from Brazil. Scientia Agricola, v. 50, n. 3, 1993. DOI: 10.1590/S0103-90161993000300023.

HUI, Y. H. (Ed.) Bailey's industrial oil \& fat products: edible oil \& fat products application. v.1. 5 ed. Nova York: John Wiley \& Sons, 1996.

INHAMUNS, A. J.; FRANCO, M. R. B. Composition of total, neutral and phospholipids in Mapará (Hypophthalmus sp.) from the Brazilian Amazonian area. Journal of Agricultural and Food Chemistry, v. 49, n. 10, p. 4859-63. 2001. DOI: $10.1021 /$ jf0100393.

KRAUSS, R. M., ECKEL, R. H.; HOWARD, B.; APPEL, L. J.; DANIELS, S. R.; DECKELBAUM, R. J.; ERDMAN, J. W.; KRIS-ETHERTON, P.; GOLDBERG, I. J.; KOTCHEN, T. A.; LICHTENSTEIN, A. H.; MITCH, W. E.; MULLIS, R.; ROBINSON, K.; WYLIE-ROSETT, J.; ST. JEOR, S.; SUTTIE, J.; TRIBBLE, D. L.; BAZZARRE, T. L. AHA Dietary Guidelines: Revision 2000: A Statement for Healthcare Professionals From the Nutrition Committee of the American Heart Association. Circulation, v. 102, p. 2284-2299, 2000.

KRIS-ETHERTON, P. M.; HARRIS, W. S.; APPEL, L. J. Fish consumption, fish oil, omega-3 fatty acids and cardiovascular disease. Circulation, v. 106, p. 2747-2757, 2002. DOI:10.1016/0140-6736(91)91846-M.

LANDS, W. E. M. Fish, Omega-3 and Human Health. 2 ed. Champaign: AOCS Press, 2005.

LARSEN, E. P.; HELDBO, J.; JESPERSEN, C. M.; NIELSEN, J. Development of a standard for quality assessment on fish for human consumption. In: HUSS, H. H.; JACOBSEN, M.; LISTON, J. (Eds). Quality assurance in the fish industry. Proceedings of an international conference. Copenhagen, 1991. p. 351-358.

MOREIRA, A. B.; VISENTAINER, N. E. S.; MATSUSHITA, M., Fatty acids profile and cholesterol contents of three Brazilian Brycon freshwater fishes. Journal of Food Composition and Analysis, v. 14, p. 565-574, 2001. DOI: 10.1006/jfca.2001.1025.

NELSON, D. L; COX, M. L. Lehninger: Principles of biochemistry. 4 ed. New York: W. H. Freeman and Company, 2005.

NETTLETON, J. A. Omega-3 fatty acids and health. New York: Thomson, 1995.

OGAWA, M.; MAIA, E. L. Manual de pesca: ciência e tecnologia do pescado. São Paulo: Livraria Varela, 1999.

OLIVEIRA, E. R. N.; AGOSTINHO, A. A.; MATSUSHITA, M. Effect of biological variables and capture period on the proximate composition and fatty acid composition of dorsal muscle tissue of Hypophtalmus edentates (Spix, 1829). Brazilian Archives of Biology and Technology, v. 46, n. 1, p. 105-114, 2003. DOI: 10.1590/S151689132003000100015 .

RAMOS FILHO, M. M.; RAMOS, M. I. L.; HIANE, P. A.; SOUZA, E. M. T. Perfil lipídico de quatro espécies de peixes da região pantaneira de Mato Grosso do Sul. Ciência e Tecnologia de Alimentos, Campinas, v. 28, n. 2, p. 361365, 2008. DOI: 10.1590/S0101-20612008000200014. 
RUEDA, F. M.; HERNÁNDEZ, M. D.; EGEA, M. A.; AGUADO, F.; GARCÍA, B.; MARTÍNEZ, F. J. Differences in tissue fatty acid composition between reared and wild sharpsnout sea bream, Diplodus puntazzo (Cetti, 1777). British Journal of Nutrition, n. 86,v. 05, p. 617-622, 2001. DOI: 10.1079/BJN2001438.

SALO-VÄÄNÄNEN, P. P.; KOIVISTOINEN, P. E. Determination of protein in foods: comparison of net protein and crude protein $(\mathrm{N} \times 6.25)$ values. Food Chemistry, Londres, v. 57, n. 1, p. 27-31, 1996. DOI: 10.1016/03088146(96)00157-4.

SANCHES, L. Pescado: matéria-prima e processamento. Fundação Cargil, Campinas, 1989. 61 p.

SANTOS-SILVA. J.; BESSA, R. J. B.; SANTOS-SILVA, F. Effects of genotype, feeding system and slaughter weigt on the quality of light lambs. II. Fatty acid composition of meat. Livestock Production Science, v. 77, n. 2-3. p. 187194, 2002. DOI: 10.1016/S0301-6226(02)00059-3.

SHAHIDI, F.; FINLEY, J. W. Omega-3 fatty acids: chemistry, nutrition and health effects. Washington: American Chemical Society, 2001.

SIKORSKI, E. Z. Tecnologia de los productos del mar: recursos, composicion nutritiva y conservacion. Zaragoza: Acribia, 1994.

SOUZA, R. F. C.; IVO, C. T. C.; SOUZA, R. A. Análise da Estrutura de Comprimento e Biometria do Pargo, Lutjanus purpureus (Poey, 1875), Capturado na Plataforma Continental da Região Norte do Brasil. Boletim Técnico-Científico do CEPNOR, Belém, v. 6, p. 61-78, 2006.

STAR 6.0. Star chromatography workstation, Varian Inc. version 6.0, 2003.

STATISTICA for Windows 5. 5. Computer program manual. Tulsa: StatSoft, Inc., 2000.

SUÁREZ-MAHECHA, H.; FRANCISCO, A.; BEIRÃO, L. H.; BLOCK, J. M.; SACCOL, A.; PARDO-CARRASCO, S. Importância de ácidos graxos polinsaturados presentes em peixes de cultivo e de ambiente natural para a nutrição humana. Boletim do Instituto de Pesca, São Paulo, v. 281, n. 1, p. 101-110, 2002.

SUGANO, M.; HIRAHARA, F. Polyunsaturated fatty acids in the food chain in Japan. American Journal of Clinical Nutrition, New York, v. 71, n. 1, sup., 189S-96S, 2000.

ULBRICHT, T. L. V.; SOUTHGATE, D. A. T. Coronary heart disease: seven dietary factors. Lancet. Londres, v. 338, n. 8773, p. 985-992, 1991. DOI: doi:10.1016/0140-6736(91)91846-M

VISENTAINER, J. V.; NOFFS, M. D.; CARVALHO, P. O.; ALMEIDA, V. V.; OLIVEIRA, C. C.; SOUZA, N. E. Lipid content and fatty acid composition of 15 marine fish species from the southeast coast of Brazil. Journal of the American Oil Chemists' Society, v. 84, p. 543-547, 2007. DOI: 10.1007/s11746-007-1070-4.

WILLIAMS, C. H. Dietary fatty acids and human health. Annales de zootechnie, Versailles, v. 49, 165-180, 2000.

\section{Dados dos autores}

Nome completo: Hugo Antonio Lima de Souza

Filiação institucional: Universidade Estadual de Campinas - Unicamp

Departamento: Departamento de Ciência de Alimentos

Função ou cargo ocupado: Doutorando em Ciência de Alimentos

Titulação: Mestre em Ciência e Tecnologia de Alimentos

Endereço: Universidade Estadual de Campinas, Faculdade de Engenharia de Alimentos. Rua Monteiro Lobato, 80, Barão Geraldo, CEP 13083-862, Campinas, São Paulo, Brasil.

Telefone para contato: (19) 3521-2159

e-mail: hugolima@fea.unicamp.br

Nome completo: Ádria de Sousa Bentes

Filiação institucional: Universidade Estadual de Campinas - Unicamp

Departamento: Departamento de Ciência de Alimentos 
Função ou cargo ocupado: Doutoranda em Ciência de Alimentos

Titulação: Mestre em Ciência e Tecnologia de Alimentos

Endereço: Universidade Estadual de Campinas, Faculdade de Engenharia de Alimentos. Rua Monteiro Lobato, 80, Barão Geraldo, CEP 13083-862, Campinas, São Paulo, Brasil.

Telefone: (19) 3521-2159

e-mail: adriasb@fea.unicamp.br

Nome completo: Marilda Garcia Simões

Filiação institucional: Universidade do Estado do Pará - UEPA

Departamento: Departamento de Tecnologia de Alimentos

Função ou cargo ocupado: Professora Assistente II

Titulação: Mestre em Alimentos e Nutrição

Endereço: Centro de Ciência Naturais e Tecnologia - CCNT, Tv. Éneas Pinheiro 2626, Bairro Marco, CEP 66095-100, Belém, Pará, Brasil.

Telefone: (91) 3276-9511, Ramal: 204

e-mail: marildags@uol.com.br

Nome completo: Mauro José Pantoja Fontelles

Filiação institucional: Universidade da Amazônia - Unama

Departamento: Pró Reitoria de Pesquisa, Pós Graduação e Extensão

Função ou cargo ocupado: Professor Titular

Titulação: Doutor em Cirurgia

Endereço: Universidade da Amazônia, Pró Reitoria de Pesquisa, Pós Graduação e Extensão, Superintendência de Pesquisa. Rua Alcindo Cacela, 287, Bairro Umarizal, CEP 66060-000, Belem, Pará, Brasil.

Telefone: (91) 40093120

e-mail: mfontelles@bol.com.br 